\title{
Vibrational Heat Transfer Intensification in an Annulus with a Deformable Boundary
}

\author{
Nikolai Kozlov iD \\ Hydrodynamic Stability Lab., Institute of Continuous Media Mechanics UrB RAS, PFRC UrB RAS, Perm, Russia \\ Correspondence should be addressed to Nikolai Kozlov; kozlov.n@icmm.ru
}

Received 30 November 2018; Revised 24 April 2019; Accepted 15 May 2019; Published 16 June 2019

Academic Editor: Matteo Aureli

Copyright (c) 2019 Nikolai Kozlov. This is an open access article distributed under the Creative Commons Attribution License, which permits unrestricted use, distribution, and reproduction in any medium, provided the original work is properly cited.

An experimental study is carried out on heat transfer in a horizontal liquid annulus at boundary oscillations. The inner boundary is formed by a copper rod that is heated using electric current. The outer boundary is made of an elastic silicone tube brought to oscillations by two electrodynamic vibrators situated symmetrically on the opposite sides. In the absence of vibrations, a classic natural convection is observed. Vibrations excite steady time-average flows in the fluid. The steady flows make the gravity-induced flow structure transform, and the heat transfer is intensified. In the analysis, it is shown that the heat transfer in the studied system is determined by a competition between the natural convection and steady streaming. A domain of parameters is found where the latter dominates.

\section{Introduction}

Heat and mass transfer is a key process for many modern technologies. The transfer rate is often a parameter on which the production costs depend. For this reason, the possibility of heat and mass transfer intensification is an actual challenge for modern industries and research. Vibrations can generate new steady states of complex mechanical systems [1] and are successfully applied in modern technologies. One of the vibrational effects that is often encountered in hydromechanical systems is steady streaming [2].

Steady streaming is a very actual problem for fluid motion in closed oscillating containers, and it is an efficient mean of mass transfer intensification. For example, in [3-5], the excitation of steady streaming in liquid droplets was achieved by acoustic levitation. Oscillations of a droplet immersed in the surrounding liquid have a potential for application in liquidliquid extraction [6]. Surfactant accumulation on the droplet interface leads to stagnation [7] that vibrational mixing can potentially prevent. Theoretical studies $[8,9]$ demonstrate that steady flows in an oscillating droplet have the shape of toroidal vortices and lead to the interface renewal by tangential time-average flows. Recent experiments $[10,11]$ on fluid oscillations in cylindrical and spherical containers with elastic boundaries demonstrate that similar steady flows can be modelled in relatively large containers.

The aim of the present study is to investigate the relation between steady streaming and heat and mass transfer. Previously, Ivanova et al. [12] performed an original experiment where the steady streaming (or forced vibrational convection) was generated by an oscillating disk. They showed that the vibrational mixing produces an efficient heat transfer and allows achievement of homogeneous temperature distribution in the fluid volume. For a model in the present study, a nonisothermal horizontal coaxial layer is chosen. In a horizontal annulus with a hot inner cylinder, natural thermal convection develops in the form of two symmetric rolls and a hot plume in the region of their contact, above the inner cylinder [13]. If the annulus is vibrated as a whole in the fluid, the thermal vibrational convection develops, which alters the fluid motion, and enhances the heat transfer [14].

In the present experiments, we consider an annulus between two horizontal concentric cylinders filled with a viscous fluid. The inner cylinder is rigid and hot. The outer cylinder is elastic, and it is kept at a constant temperature via water cooling and brought into oscillations by external electrodynamic actuators. The average boundary shape is virtually circular. The goal of the work is to study the heat 
transfer from the inner cylinder to the fluid in the cooling jacket under the condition of oscillating outer boundary. The dimensionless frequency of vibrations, which determines the squared ratio between the characteristic and the viscous lengths, is set relatively low, so as to be of interest for the application to droplet-size systems (see the argumentation in [10]). The amplitude parameter is varied in a sufficiently large range. In the analysis of experimental results, a comparison of different vibratory mechanisms is done.

\section{Experimental Setup and Technique}

In the experiments, a cylindrical annulus was considered whose outer boundary was made of an elastic silicone tube (1) (Figures 1(a) and 1(b)) and the inner boundary was formed by a copper cylindrical heat exchanger (2). On the outer boundary, two rectangular activators (3) were installed symmetrically. The radius of the copper cylinder was $R_{1}=7.5 \mathrm{~mm}$ and its length $l_{h}=194 \mathrm{~mm}$. The interior dimensions of the silicone cylinder were $R_{2}=25.0 \mathrm{~mm}$ and $L=200.0 \mathrm{~mm}$, and the thickness of the walls was $2 \mathrm{~mm}$. The annulus gap was $h=R_{2}-R_{1}=17.5 \mathrm{~mm}$. The two cylinders were fixed on transparent flanges (4) and sealed hermetically. The copper cylinder was slightly shorter than the working volume in order to avoid heat sinking through the front and rear flanges, and it was fixed equidistantly from the flanges with the help of small plastic cylinders. Each activator consisted of two aluminium plates with length $l=170.0 \mathrm{~mm}$, height $d=20.0 \mathrm{~mm}$, and thickness $1.2 \mathrm{~mm}$. The plates squeezed the silicone wall in a sandwich-like configuration (Figure 1(b)).

The model was placed in a water-cooling jacket (5), integrated with the flanges, that maintained a constant temperature $T_{2}$ of the outer boundary by means of pumping water from a thermostatic bath trough openings $(6,7)$. To provide temperature $T_{1}$ of the inner cylinder, a nichrome wire was installed in a shaft in the middle of the heat exchanger, and it was powered by a DC supply (8). The heating power $Q$ was calculated with the values of current $I$ and voltage $U$ measured from the indicators of the power supply, $Q=I U$. The temperature $T_{2}$ was controlled by two thermistors (9) of Pt100 type installed in the front and the rear sides of the jacket. The temperature difference between the inner and outer boundaries of the annulus, $\Delta T=T_{1}-T_{2}$, was measured directly by a differential thermocouple of type $T$ (copperconstantan). Its hot junction (10) was put in a narrow groove in the heat exchanger, while the cold junction (11) was immersed in cooling water. The thermometers were connected to a multichannel measuring device Termodat 22K5 (12) (Figure 1(c)). Temperature was measured with an accuracy of $0.1^{\circ} \mathrm{C}$. The discrepancy between the data from the front and the rear thermistors did not exceed the experimental error, which allows us to conclude that the water in the cooling jacket had a uniform temperature distribution.

The annulus was filled through an opening (13) in the rear flange with a working fluid, an aqueous solution of glycerol with a concentration of 0.90 was used. The corresponding kinematic viscosities varied in the range $\nu=(0.73-1.90)$ St depending on temperature. The thermal diffusivity was $\chi=(9.7-9.9) \cdot 10^{-4} \mathrm{~cm}^{2} / \mathrm{s}$.
The activators were mechanically connected with the rods of electromagnetic actuators Dunkermotoren STA1112 (14) (Figure 1(c)). Each actuator was controlled by an amplifier Copley Accelnet ACJ-055-18-S and fed by a DC power supply MASTECH HY5020E. The position of the actuators was defined by a signal from a generator GSPF-052 integrated in a computer. The actuators performed synchronous periodic motion and induced vibrations of the activators. As a result, the elastic boundary was periodically contracted and extended along the horizontal axis as shown in Figure 1(a). The vibration frequency was set at $f=3.00 \mathrm{~Hz}$, and the amplitude varied in the range $b=(0-11) \mathrm{mm}$. The oscillations of the opposite activators were symmetric, and the oscillation amplitude of the whole boundary was equal to the span of one activator (Figure 1(a)). In the experiments, $b$ was found as an average of the values obtained for the left and the right activators, the discrepancy between them did not exceed $0.1 \mathrm{~mm}$.

In order to study the flow structure, the working fluid was seeded with Rilsan light-scattering powder, characterised by particle size of $40 \mu \mathrm{m}$ and density of $1.03 \mathrm{gr} / \mathrm{cm}^{3}$. A transversal cross section of the annulus was illuminated with a light-sheet from a solid-state continuous laser (15) (Figure 1(c)). The time-average flow structure and velocity were studied using PIV (particle image velocimetry) by the means of the software PIVlab [15]. The images were taken all in the same oscillation phase with a timestep being a multiple of the oscillation period. This way, the time-average velocity was calculated directly. Before the experiments, test runs were performed, which demonstrated that the flow structure reproduced itself in different cross sections along the cylinder axis, except the domains near the flanges. Hence, within the considered range of experimental parameters, the steady flows under the study may be considered effectively two-dimensional.

The sequence of an experiment was as follows. First, the thermostatic bath was set up running until the fluid in the cooling jacket achieved its working temperature, $T_{2}=20.0 \pm 0.1^{\circ} \mathrm{C}$. Then, $T_{2}$ remained constant during the whole experiment. Second, the heating power $Q$ was adjusted to a constant value, and, depending on $Q$, it took up to an hour for the system to achieve equilibrium. At that moment, a temperature difference $\Delta T_{0}$ settled, which corresponded to the case of natural convection, in the absence of vibrations. The system was maintained at $\Delta T_{0}$ for more than 15 minutes. Then the vibrations were initiated, and their amplitude was increased step by step. On each step, the system needed about 15 minutes to achieve a constant value of $\Delta T$, and then it was maintained at this temperature for 15-20 minutes while the measurements were done. The temperature values were registered continuously during the entire experiment and processed afterward. Every value of $\Delta T$ was averaged over more than 100 instantaneous values. Experiments were then repeated at different $Q$.

\section{Experimental Results}

In the absence of vibrations, due to homogeneous heating along the length of the inner cylinder in the annulus, convection starts. The flow consists of two mirror symmetric 

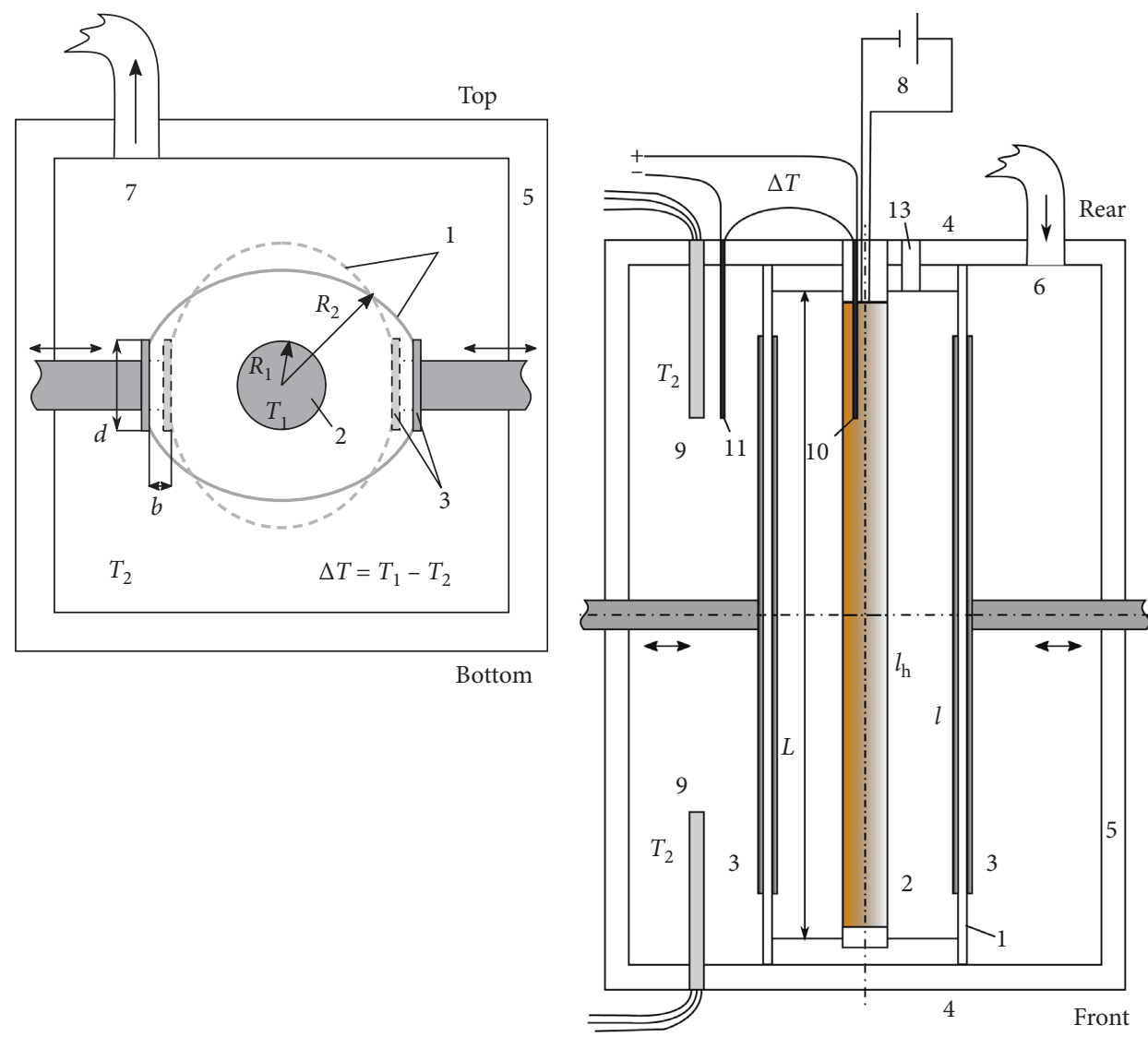

(a)

(b)

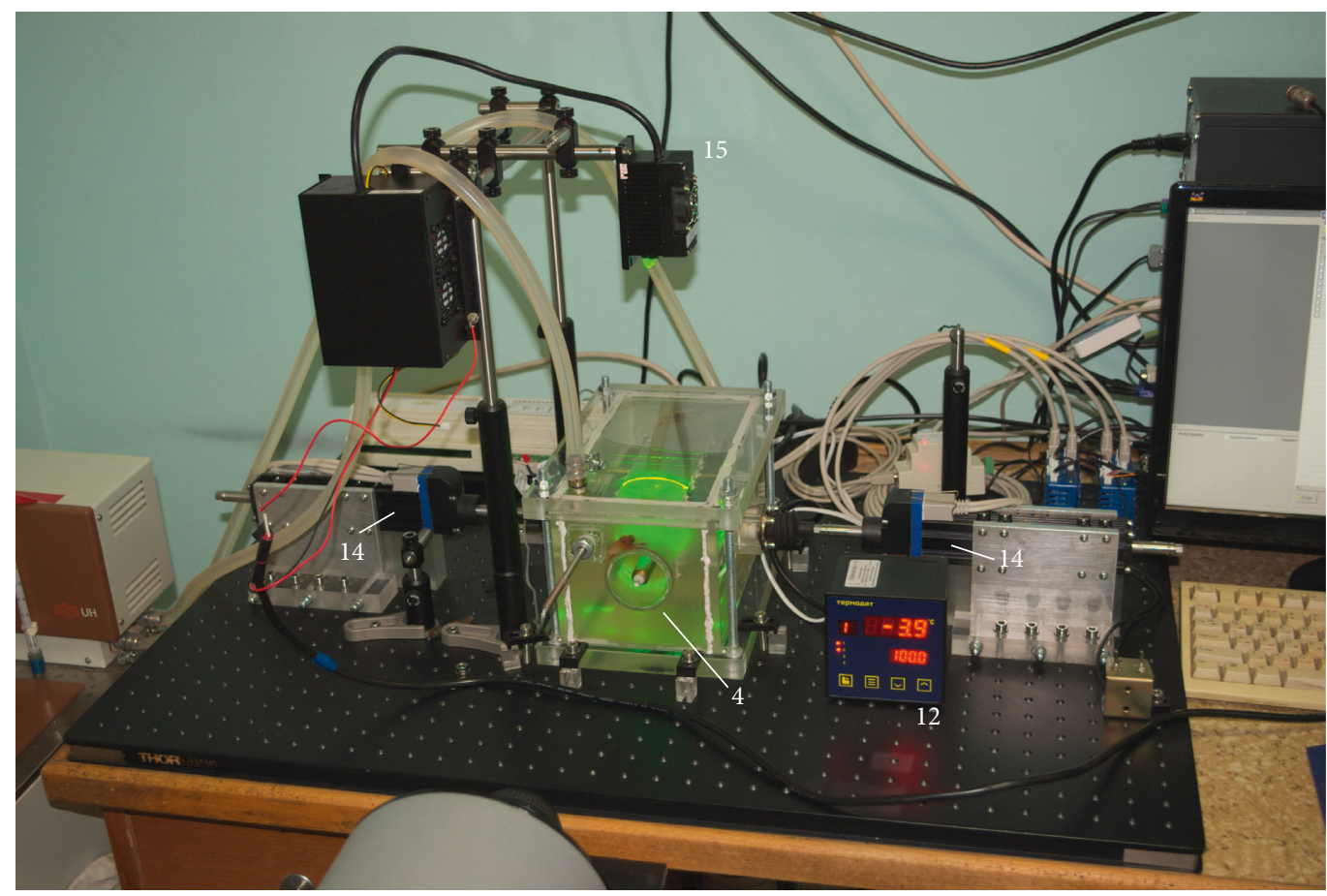

(c)

Figure 1: Experimental setup: schematic (not up to scale) of the experimental cell ((a) front view; (b) top view); photograph of the setup ((c) 1-elastic boundary; 2-heat exchanger; 3-activators; 4-transparent flanges; 5-cooling jacket; 6-cooling water inlet; 7-outlet; 8-power supply; 9-thermistors; 10, 11-thermocouple; 12-Termodat; 13-opening; 14-actuators; 15-laser). 
rolls extended parallel to the cylinder axis (Figure 2(a)). The fluid streams upwards near the hot inner cylinder and downwards along the cold outer boundary. In the region of contact of the rolls, above the copper rod, an ascending convective plume is observed. This type of convection was studied and described in detail in many studies, e.g., [13].

In the presence of vibrations, the flow structure dramatically changes; instead of two rolls, we observe a system of multiple rolls of different sizes and intensities (Figure 2(b)). The larger rolls are distributed along the outer boundary, and the small ones around the inner cylinder. Interestingly, in some local points, the vibratory flows have the inverse direction as compared to the natural convection. In the immediate contact with the inner cylinder, six vorticity peaks are observed (Figure 2(b)), and according to the regular way of their spatial distribution, it is possible to suggest the presence of two more peaks in the shadow area. In the bottom part of the annulus, four large rolls are distributed regularly and in conjunction with the small rolls. This regular flow pattern closely resembles to those observed in isothermal oscillating droplets or containers with elastic boundary [8-11] and should be related to the steady flows due to the boundary oscillations.

The temperature difference between the inner and the outer boundaries $\Delta T$ increases with an increase in the heating power $Q$ (Figure 3). The highest $\Delta T$ values are observed in the case of natural convection (Figure 3, points 1). Vibrations lead to a decrease in the temperature difference (Figure 3, points 2-5), thus intensifying the heat transfer. The highest vibration amplitude $b$ corresponds to the smallest $\Delta T$.

\section{Analysis of Results}

4.1. Dimensionless Parameters of the Problem. Below are defined the dimensionless parameters used in the discussion.

Prandtl number

$$
\operatorname{Pr}=\frac{\nu}{\chi}
$$

Nusselt number

$$
\mathrm{Nu}=\frac{Q}{Q_{\mathrm{m}}},
$$

where $Q_{m}$ is the molecular heat flux calculated as

$$
Q_{\mathrm{m}}=\frac{2 \pi \lambda L \Delta T}{\ln \left(R_{2} / R_{1}\right)},
$$

$\lambda$ is the thermal conductivity.

Rayleigh number

$$
\mathrm{Ra}=\frac{g \beta \Delta T h^{3}}{\nu \chi},
$$

where $h=R_{2}-R_{1}$ is the annulus thickness, $g$ is the gravity acceleration, $\beta$ is the thermal expansion coefficient.

Vibrational parameter

$$
R_{v}=\frac{(b \Omega \beta \Delta T h)^{2}}{2 \nu \chi} .
$$

where $\Omega=2 \pi f$ is the angular frequency of vibrations.

Vibrational Grashof number

$$
\mathrm{Gr}_{v}=\frac{b^{2} \Omega^{2} h^{2} \beta \Delta T}{v^{2}}
$$

Dimensionless frequency

$$
\omega=\frac{\Omega h^{2}}{v} .
$$

Pulsation Reynolds number

$$
\operatorname{Re}_{\mathrm{p}}=\frac{b^{2} \Omega}{v} .
$$

4.2. Heat Transfer without Vibration. The problem of natural convection in an annulus with the hot inner cylinder has been studied by many authors in a wide range of $\mathrm{Ra}, \mathrm{Pr}$, and $R_{2} / R_{1}$. According to correlations based upon experimental data by different authors, in the range of parameters $\mathrm{Ra}=10^{3}-10^{8}, \operatorname{Pr}=1-10^{3}$, and $R_{2} / R_{1}=1.2-3.3$, the heat transfer by convection may be described by the scaling law $\mathrm{Nu} \sim \mathrm{Ra}^{0.25}[16-18]$.

In [16], for the correlation, the Rayleigh number was calculated with a modified layer thickness $h_{\mathrm{m}}=\left(R_{1} \cdot R_{2}\right)^{1 / 2}$. $\ln \left(R_{2} / R_{1}\right)$,

$$
\mathrm{Ra}_{\mathrm{m}}=\frac{g \beta \Delta T h_{\mathrm{m}}^{3}}{\nu \chi} .
$$

The corresponding dependence reads as follows [16]:

$$
\mathrm{Nu}=0.2 \mathrm{Ra}_{\mathrm{m}}^{0.25} \text {. }
$$

The results of the present experiments reveal a dependence that is close to (10), but does not exactly match it (Figure 4). The possible reasons for this discrepancy might be the following: in the present experiments, the annulus is not strictly uniform in width because of the activators (Figure 1(a)), and the latter may also create an inhomogeneity in the temperature boundary conditions on the outer wall. On the whole, the obtained results witness that we observe the classical convection in the annulus with the hot inner boundary and allow for the further analysis in a more complicated situation with the vibrating outer boundary.

4.3. Heat Transfer at Vibration. According to [19], two situations should be distinguished. In the first case, the fluid moves with the container as a solid body. When the temperature field in the fluid bulk is nonhomogeneous, the vibration field excited by oscillations of the container as a whole acts on the fluid-density inhomogeneities induced by nonuniform temperature distribution. In the second case, boundary conditions are nonuniform, and this leads to the formation of a nonuniform pulsation velocity field. Then, fluid motion relative to the cavity exists even if the temperature distribution is uniform. Also in this situation, vibrations excite fluid motion when the container is immobile and only a part of it (e.g.,a deformable wall) oscillates. 


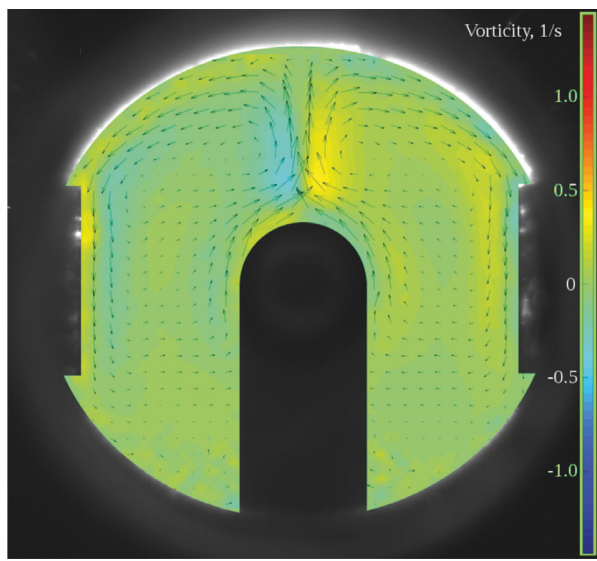

(a)

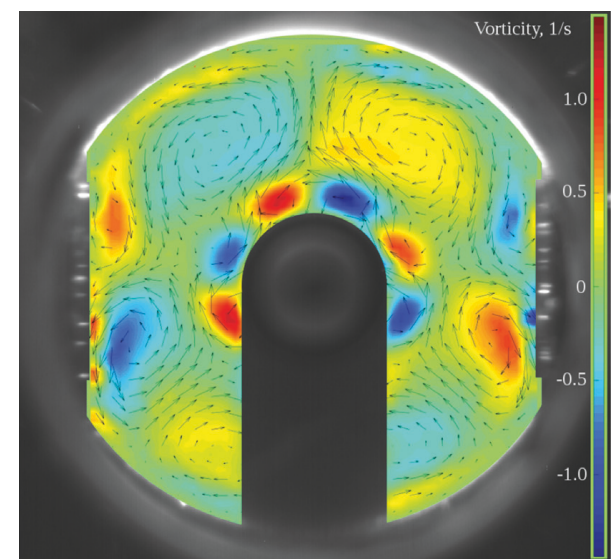

(b)

FIGURE 2: Vector plots of steady-flow velocity fields at the heating power $Q=27.2 \mathrm{~W}$ in the absence of vibrations (a) and at vibrations with the frequency $f=3.00 \mathrm{~Hz}$ and the amplitude $b=10.2 \mathrm{~mm}(b / h=0.58)$. (b) The colour maps represent vorticity calculated from the vector field of the time-average steady velocity. Below the inner cylinder, a shadow is seen.

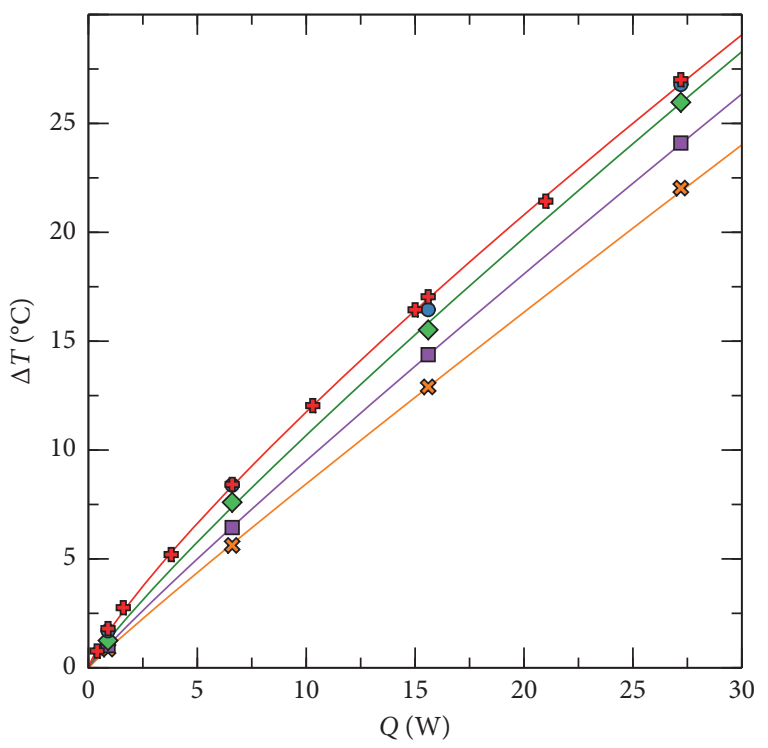

$b / h$

$\begin{array}{ll}\text { (1) } 0 & \text { (4) } 0.41 \\ \circ(2) 0.17 & \text { « (5) } 0.53 \\ \diamond(3) 0.29 & \end{array}$

Figure 3: The temperature difference $\Delta T$ versus the heating power $Q$ in the case of natural convection (1) and at vibrations with the amplitude $b=3.0$ (2), 5.1 (3), $7.2(4)$, and $9.2 \mathrm{~mm}(5)$.

In the first case (see [19], Chapters 1-5), the basic equations of thermovibrational convection, obtained by averaging, include three dimensionless parameters: $\mathrm{Ra}, R_{v}$, and Pr. The dynamics of a nonisothermal system is then determined by the relative contribution of the natural convection $(\mathrm{Ra})$ and the thermovibrational convection $\left(R_{v}\right)$. An example to such situation is vibrations of the horizontal annulus with the hot inner boundary, while both boundaries are rigid $[14,20]$. In the second case (see [19], Chapters 6-9), the equations contain $\mathrm{Re}_{\mathrm{p}}, \mathrm{Pr}$, and $\mathrm{Gr}_{v}$. The latter may be rewritten as $\mathrm{Gr}_{v}=\mathrm{Re}_{\mathrm{p}} \omega \beta \Delta T$. Here, $\mathrm{Re}_{\mathrm{p}}$ characterises the

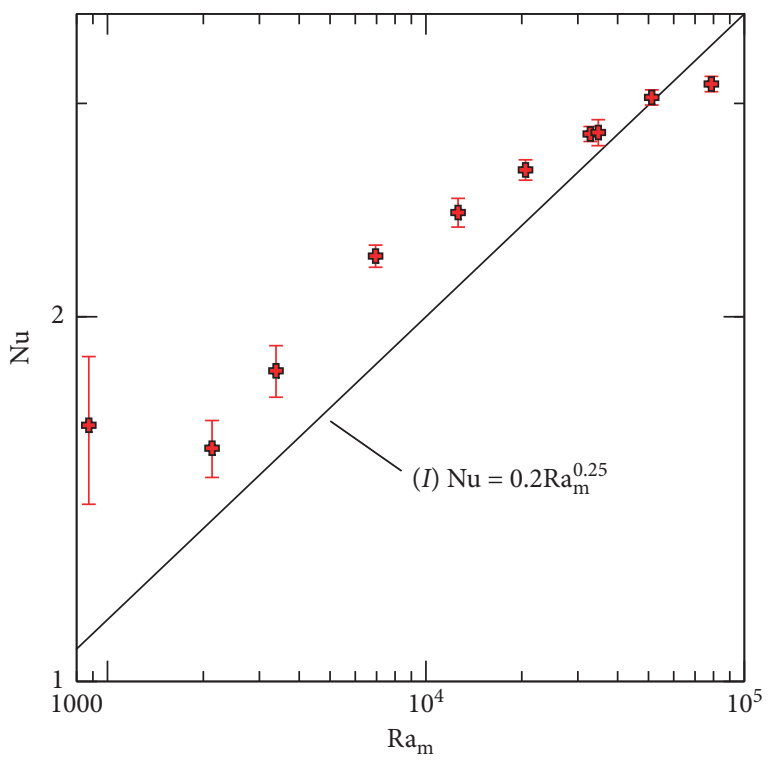

亞 1

Figure 4: The dependence of $\mathrm{Nu}$ on $\mathrm{Ra}_{\mathrm{m}}$ in the absence of vibrations. Curve $I$ is plotted according to equation (10).

intensity of the steady streaming, while $\mathrm{Gr}_{v}$ is responsible for the thermal vibrational mechanism. The present study belongs to the second case since the container is immobile and the periodic deformation of the outer boundary is the source of oscillations. In most of the present experiments, $\omega \beta \Delta T \sim 10^{-2}-10^{-1}$, and one should expect that the thermal vibrational convection will manifest itself only through the second-order effects due to the interaction between the oscillatory flow and inhomogeneous temperature field [19]. An exception is experiments at $\mathrm{Q}=27.2 \mathrm{~W}$, where $\omega \beta \Delta T \lesssim 1$.

Forced vibrational convection (steady streaming) is excited through an isothermal mechanism due to an inhomogeneity, spatial or phasic, in the amplitude of the pulsation velocity $[21,22]$. In addition to $\mathrm{Re}_{\mathrm{p}}$, it is 
characterised by $\omega$, which determines the thickness of the viscous boundary layers (Stokes layers). Two limiting situations are possible. First, the high-frequency limit that is formally defined as $\omega \gg 1$ : this means that the oscillating flow may be considered potential outside thin boundary layers on the walls. Second, the low-frequency limit, $\omega \sim 1$ : this means that viscous force is important to the oscillating flow in the entire container volume. As shown in $[10,11]$, with an increase in $\omega$, the steady-flow structure transforms.

In the high-frequency limit, outside the boundary layers, a flow exists, whose velocity in the direction tangential to the boundary is as follows [22]:

$$
v_{\tau}=-\frac{3}{4 \Omega} V_{0} \frac{\partial V_{0}}{\partial x_{\tau}}
$$

Taking into account that $V_{0}=b \Omega$ and nondimensionalizing the velocity by $v / h$, from (11), one obtains

$$
\frac{v_{\tau} h}{v} \sim \frac{b^{2} \Omega}{v} \sim \operatorname{Re}_{\mathrm{p}} .
$$

This gives the meaning of $\mathrm{Re}_{\mathrm{p}}$ : it determines the dimensionless velocity of steady streaming. The latter is independent of the dimensionless frequency. In the lowfrequency limit, as shown theoretically in [11], the parameter that determines the magnitude of the dimensionless velocity of the steady flows is $\operatorname{Re}_{\mathrm{p}} \omega=b^{2} \Omega^{2} h^{2} / v^{2}$, where $h$ is the characteristic size of the container. Both limiting cases are confirmed experimentally in containers with deformable oscillating boundaries $[10,11]$.

The analysis of dimensionless parameters shows that in the present experiments, vibrations have two principal effects on the heat transfer. First, they lead to an increase of $\mathrm{Nu}$, as one may see by comparing experimental results at different vibration amplitudes shown in Figure 5. Second, they reduce dramatically the rate of $\mathrm{Nu}$ growth with $\mathrm{Ra}$, so for the highest amplitude (points 5) the index of power is an order of magnitude lower than for the case without vibrations (points 1). Contrary to this, in the case of thermal vibrational convection excited by vibrations of the entire container, vibrations lead to an increase of the rate of $\mathrm{Nu}$ growth with $\mathrm{Ra}$ [14]. Obviously, the effect of vibrations on the $\mathrm{Nu}(\mathrm{Ra})$ dependence is opposite in the two cases. This fact clearly demonstrates that the vibrations influence the system behaviour through a different mechanism (steady streaming, as it will be demonstrated below), and the thermal vibrational mechanism is secondary in the present study. The approximating curves on the graph approach each other with the increase in the Rayleigh number, this means that the relative effect of vibrations is maximal in the region of smallest Ra.

The intensity of steady streaming at $\omega=$ const is determined by the pulsation Reynolds number $\operatorname{Re}_{\mathrm{p}}$ [19]. The fluid viscosity has a pronounced dependence on the temperature. For this reason, despite all experiments conducted at the constant vibration frequency, the dimensionless frequency varies in the range $\omega=30-80$. According to $[10,11]$, where cylindrical and spherical containers with oscillating boundaries were considered, in this range, the

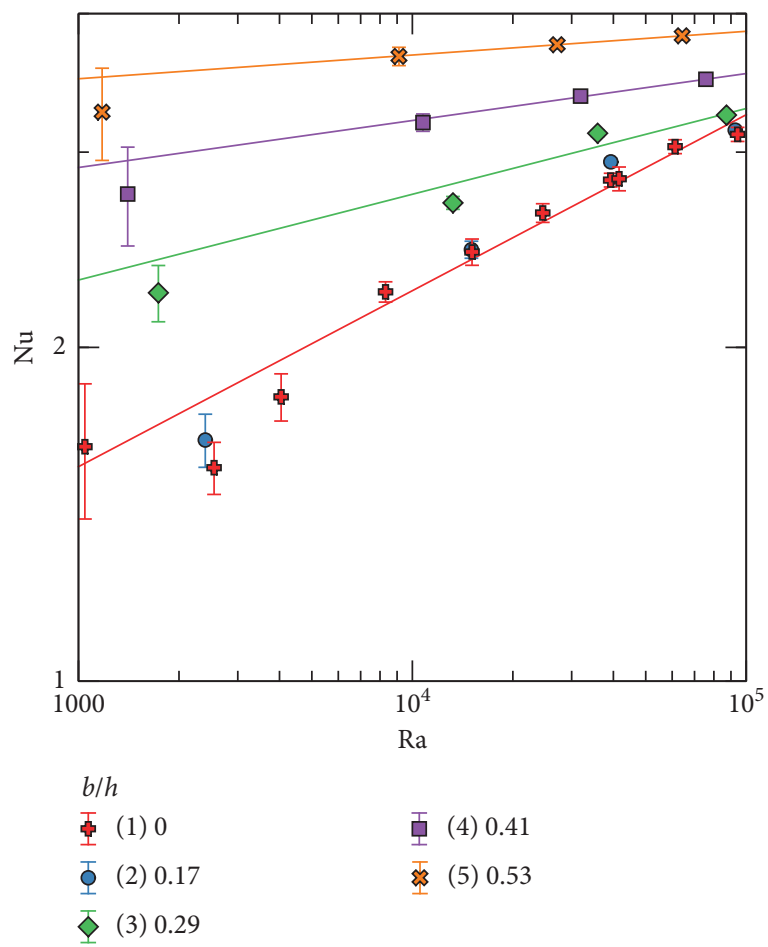

FIgURE 5: The dependence of $\mathrm{Nu}$ on the convective parameter Ra. The experimental series correspond to the following ranges of $\omega$ : 0 (1), $32.3-78.4$ (2), $31.8-76.2$ (3), $31.5-71.3$ (4), and $31.4-66.3$ (5).

dependence on $\omega$ was weak. Thus, one may assume that, for the presently studied system, the main parameter that controls the steady streaming is indeed $\mathrm{Re}_{\mathrm{p}}$.

Let us analyse the dependence of $\mathrm{Nu}$ on $\mathrm{Re}_{\mathrm{p}}$ (Figure 6). On the graph, each experimental series has two regions. In the first one, at lower $\mathrm{Re}_{\mathrm{p}}$, the vibrations do not produce any effect on the heat transfer, and the Nusselt number is virtually equal to $\mathrm{Nu}_{0}$, the corresponding value obtained without vibrations. In the second region, the experimental points follow curve $I$ (Figure 6), which is described by the following relation:

$$
\mathrm{Nu} \sim \mathrm{Re}_{\mathrm{p}}^{0.3} .
$$

The four experimental series that differ in the values of Ra by two orders of magnitude are described by the same scaling law (13). This means that the steady streaming becomes the dominant mechanism of heat transfer under a high vibrational forcing. This result is consistent with the analysis of parameters based on theory [19] and it supports the idea that the thermal vibrational convection is not important in the considered study.

The threshold value of the pulsation Reynolds number increases with an increase in $\mathrm{Ra}$; this seems reasonable because at high Rayleigh numbers, the competition between the natural convection and the steady streaming is stronger. With the increase in $\mathrm{Re}_{\mathrm{p}}$, the steady streaming dominates over the thermal convection. At $\mathrm{Re}_{\mathrm{p}} \gtrsim 10$, all experimental series lie parallel to curve $I$; however, some scattering is observed between points $2-4$ that might be related to some 


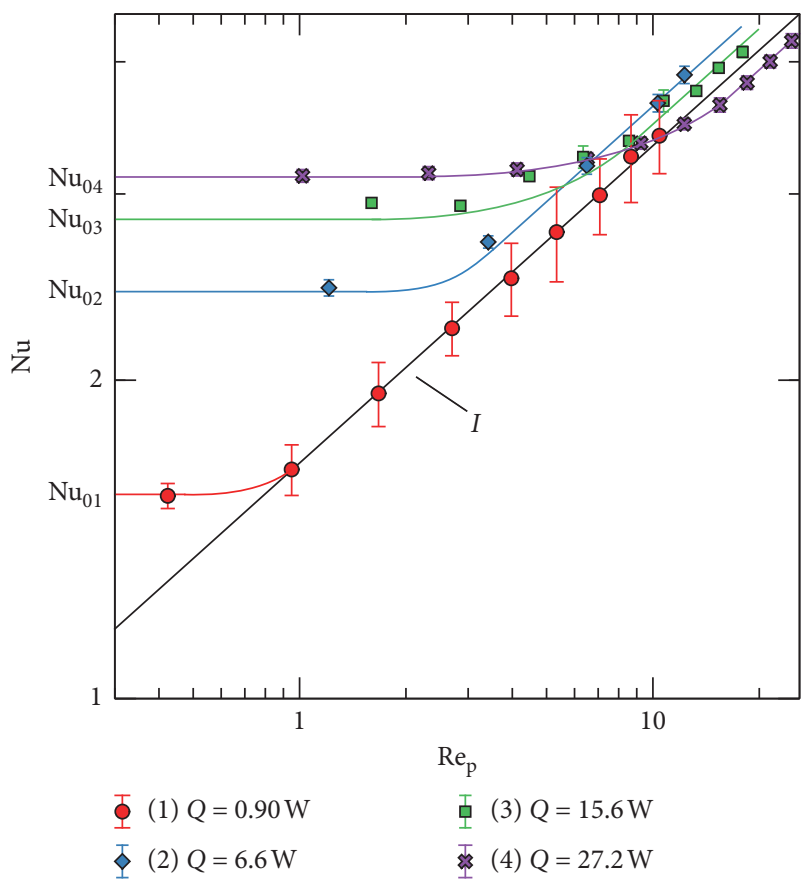

Figure 6: The dependence of $\mathrm{Nu}$ on the pulsation Reynolds number $\mathrm{Re}_{\mathrm{p}}$. The experimental series correspond to the following ranges of $\mathrm{Ra} \cdot 10^{-3}: 1.1-2.6(1), 9.1-15(2), 27-40(3)$, and $64-93$ (4), and to the following ranges of $\omega: 31.3-32.4$ (1), $36.7-41.0$ (2), $46.3-55.6$ (3), and 62.0-79.0 (4). Values of $\mathrm{Nu}_{01-04}$ are obtained at $\operatorname{Re}_{\mathrm{p}}=0$.

second-order effects mentioned in [19] and to the variation of $\omega$. At $\mathrm{Ra} \lesssim 10^{3}$, the forced convection excited by the steady streaming dominates over the natural convection in practically the entire range of considered values of $\mathrm{Re}_{\mathrm{p}}$ (points 1 ). Note that the extreme left point of series 1 corresponds to $\mathrm{Ra}=2.6 \cdot 10^{3}$.

\section{Conclusion}

An experimental investigation of heat transfer has been carried out in a fluid-filled horizontal layer enclosed between two concentric cylinders. The inner cylinder was heated and the outer one maintained at a constant temperature. The wall of the outer cylinder was elastic, and it was brought into oscillations by the means of two electrodynamic actuators situated symmetrically on the left and on the right. The thermal convection observed in the absence of vibrations is described by a scaling law that is in agreement with the correlations found in the literature. When vibrations are applied to the elastic outer boundary of the considered annulus, both the flow structure and the heat transfer rate are altered.

Due to vibrations, steady streaming is excited in the fluid. The intensity of heat transfer increases with the vibration amplitude. The analysis of dimensionless parameters shows that the thermal vibrational convection does not play role in the studied system and the governing vibrational mechanism is steady streaming. On the whole, the dynamics of the nonisothermal fluid is determined by a competition between the natural convection and the forced vibrational convection. The former dominates at small pulsation Reynolds numbers and large Rayleigh numbers, and the latter-on the contrary, at high $\mathrm{Re}_{\mathrm{p}}$ and small Ra.

In the domain $\mathrm{Ra} \leqslant 10^{3}$, the vibrational convection dominates, and the Nusselt number $\mathrm{Nu}$ is determined solely by $\mathrm{Re}_{\mathrm{p}}$. The maximal heat transfer rate enhancement due to vibrations achieved in the present experiments is about a factor of 2 .

\section{Data Availability}

The experimental data used to support the findings of this study are included within the article in the form of direct numerical values given in the text, graphs, and colour-scaled figures.

\section{Conflicts of Interest}

The author declares that there are no conflicts of interest.

\section{Acknowledgments}

This research was supported by the Russian Science Foundation (project 17-71-10189).

\section{References}

[1] I. I. Blechman, Vibrational Mechanics, Allied, New Delhi, India, 2003

[2] N. Riley, "Steady streaming," Annual Review of Fluid Mechanics, vol. 33, no. 1, pp. 43-65, 2001.

[3] E. Trinh and T. G. Wang, "Large-amplitude free and driven drop-shape oscillations: experimental observations," Journal of Fluid Mechanics, vol. 122, no. 1, pp. 315-338, 1982.

[4] A. L. Yarin, G. Brenn, O. Kastner, D. Rensink, and C. Tropea, "Evaporation of acoustically levitated droplets," Journal of Fluid Mechanics, vol. 399, pp. 151-204, 1999.

[5] A. Muginstein, M. Fichman, and C. Gutfinger, "Gas absorption into a drop in the presence of an acoustic field," International Journal of Multiphase Flow, vol. 31, no. 3, pp. 263-284, 2005.

[6] C. Hanson, Recent Advances in Liquid-Liquid Extraction, Elsevier Science, Amsterdam, Netherlands, 2013.

[7] M. Wegener, N. Paul, and M. Kraume, "Fluid dynamics and mass transfer at single droplets in liquid/liquid systems," International Journal of Heat and Mass Transfer, vol. 71, pp. 475-495, 2014.

[8] V. A. Murtsovkin and V. M. Muller, "Steady-state flows induced by oscillations of a drop with an adsorption layer," Journal of Colloid and Interface Science, vol. 151, no. 1, pp. 150-156, 1992.

[9] A. L. Yarin, "Stationary d.c. streaming due to shape oscillations of a droplet and its effect on mass transfer in liquidliquid systems," Journal of Fluid Mechanics, vol. 444, pp. 321-342, 2001.

[10] V. G. Kozlov, N. V. Kozlov, and V. D. Schipitsyn, "Steady flows in an oscillating deformable container: effect of the dimensionless frequency," Physical Review Fluids, vol. 2, no. 9, article 094501, 2017.

[11] V. G. Kozlov, R. R. Sabirov, and S. V. Subbotin, "Steady flows in an oscillating spheroidal cavity with elastic wall," Fluid Dynamics, vol. 53, no. 2, pp. 189-199, 2018. 
[12] A. A. Ivanova, V. G. Kozlov, D. A. Polezhaev, D. Pareau, and M. Stambouli, "Heat transfer in a closed cavity under conditions of forced vibrational convection," Fluid Dynamics, vol. 44, no. 4, pp. 481-489, 2009.

[13] T. H. Kuehn and R. J. Goldstein, "An experimental and theoretical study of natural convection in the annulus between horizontal concentric cylinders," Journal of Fluid Mechanics, vol. 74, no. 4, pp. 695-719, 1976.

[14] A. A. Ivanova and V. G. Kozlov, "Experimental study of the effect of vertical vibrations on convection in a horizontal cylindrical layer," Fluid Dynamics, vol. 20, no. 6, pp. 989-992, 1986.

[15] W. Thielicke and E. J. Stamhuis, "Pivlab-towards userfriendly, affordable and accurate digital particle image velocimetry in matlab," Journal of Open Research Software, vol. 2, 2014

[16] M. Itoh, T. Fujita, N. Nishiwaki, and M. Hirata, "A new method of correlating heat-transfer coefficients for natural convection in horizontal cylindrical annuli," International Journal of Heat and Mass Transfer, vol. 13, no. 8, pp. 13641368, 1970.

[17] T. H. Kuehn and R. J. Goldstein, "Correlating equations for natural convection heat transfer between horizontal circular cylinders," International Journal of Heat and Mass Transfer, vol. 19, no. 10, pp. 1127-1134, 1976.

[18] Y. T. Tsui and B. Tremblay, "On transient natural convection heat transfer in the annulus between concentric, horizontal cylinders with isothermal surfaces," International Journal of Heat and Mass Transfer, vol. 27, no. 1, pp. 103-111, 1984.

[19] G. Z. Gershuni and D. V. Lyubimov, Thermal Vibrational Convection, Wiley, Hoboken, NJ, USA, 1998.

[20] A. A. Ivanova and V. G. Kozlov, "Experimental study of the instability of convective boundary layers under the action of vibration," Fluid Dynamics, vol. 33, no. 3, pp. 324-330, 1998.

[21] G. K. Batchelor, Introduction to Fluid Dynamics, University Press, Cambridge, UK, 1967.

[22] H. Schlichting, Boundary Layer Theory, McGraw-Hill, New York, NY, USA, 1968. 


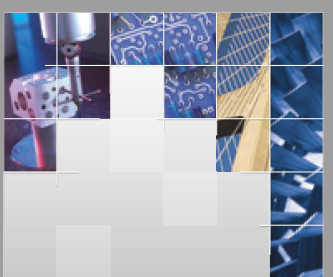

\section{Enfincering}
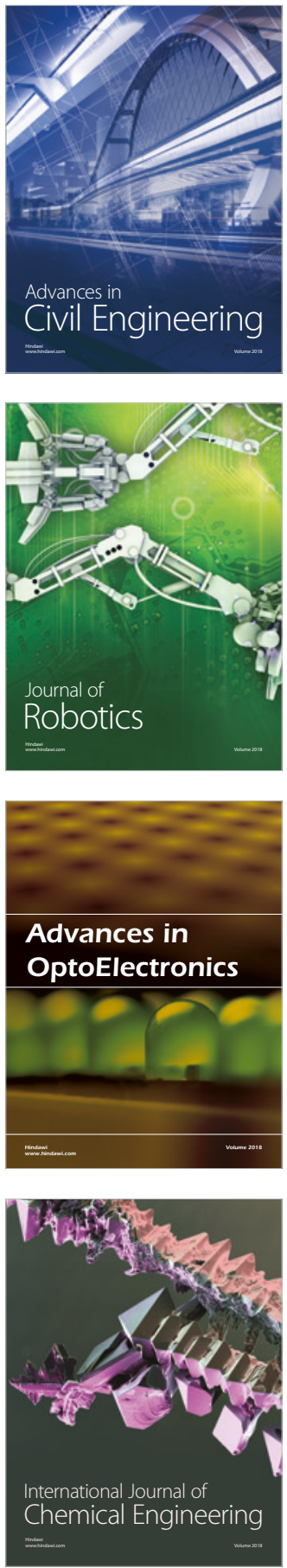

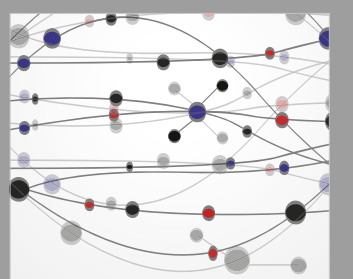

\section{Rotating \\ Machinery}

The Scientific World Journal

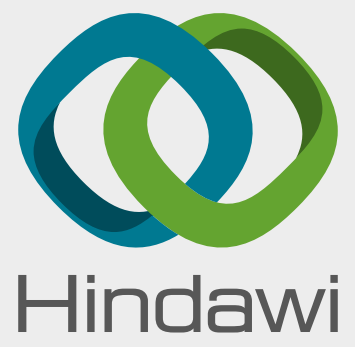

Submit your manuscripts at

www.hindawi.com
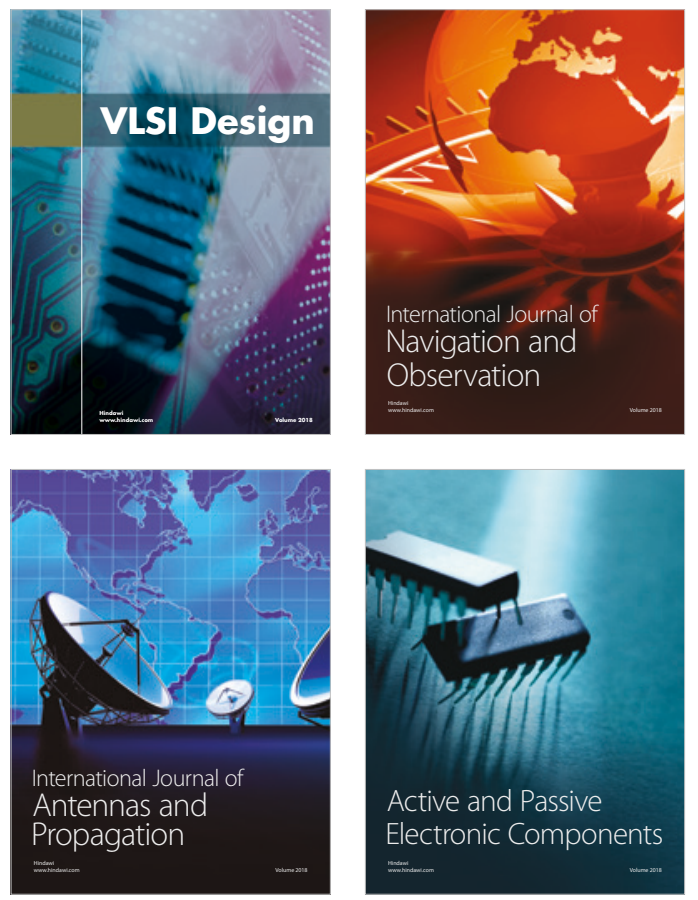
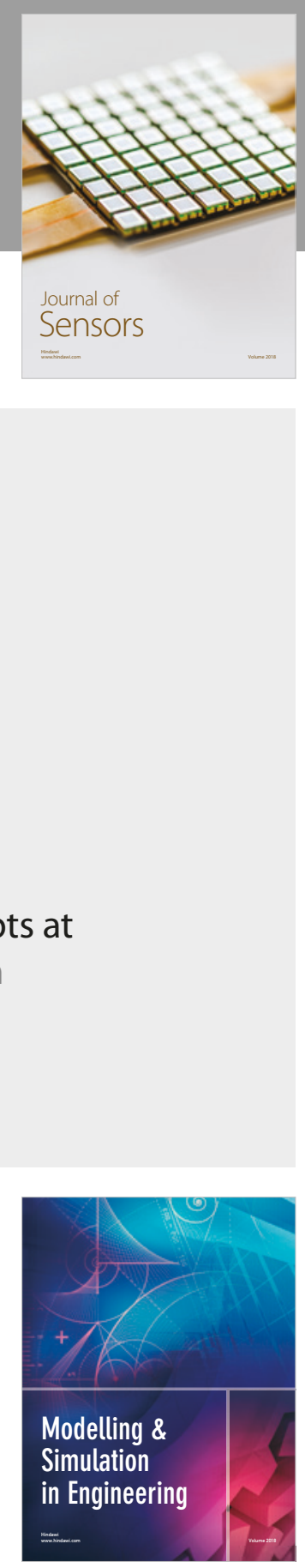

\section{Advances \\ Multimedia}
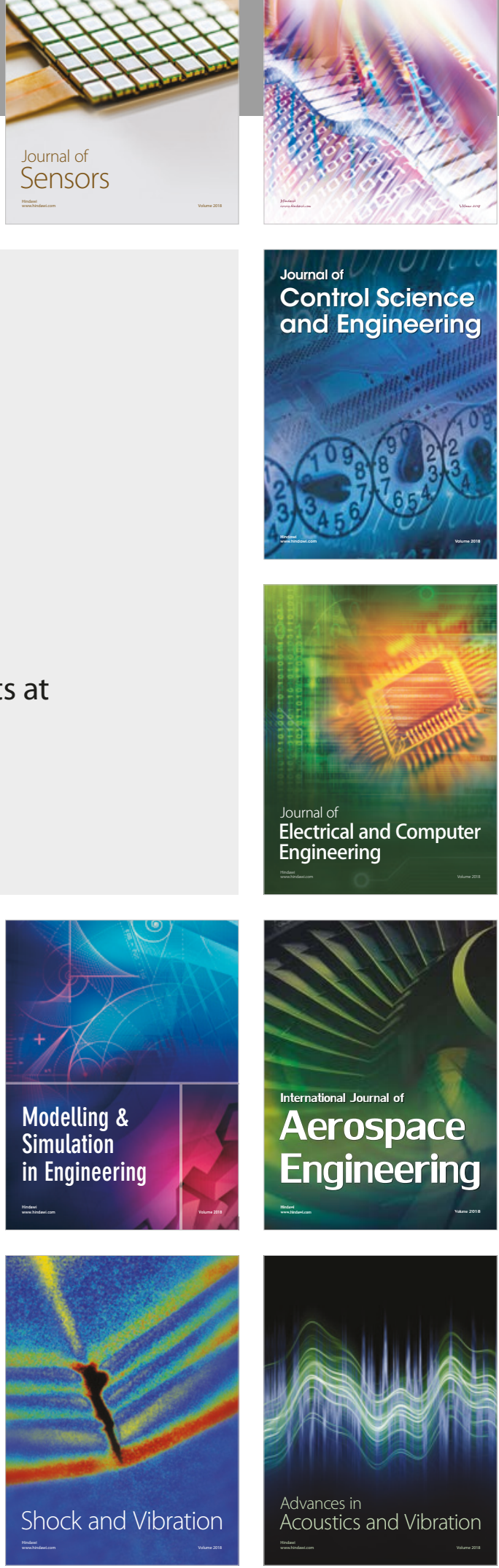